The Psychological Record, 2009, 59, 53-74

\title{
THE ROLE OF VERBAL BEHAVIOR, STIMULUS NAMEABILITY, AND FAMILIARITY ON THE EQUIVALENCE PERFORMANCES OF AUTISTIC AND NORMALLY DEVELOPING CHILDREN
}

\author{
Jennifer O’Connor \\ Applied Behaviour Analysis Centre for Autism Schooling, Kilbarrack, Ireland \\ Aoife Rafferty, Dermot Barnes-Holmes, and Yvonne Barnes-Holmes \\ National University of Ireland Maynooth
}

\begin{abstract}
The emergence of equivalence relations and the role of overall verbal competence and stimulus nameability and familiarity in this regard were investigated across 3 experiments involving 15 children diagnosed with autistic spectrum disorder (ASD), as well as 3 typically developing children. The experimental sequence comprised 4 identical stages, each with 4 phases. The relationship between verbal competence and equivalence was assessed by including children with ASD categorized with different levels of verbal behavior. Stimulus nameability and familiarity were assessed by systematically varying these features across different stimulus sets presented during 4 experimental stages. The results of all experiments indicated that the equivalence performances of both groups were influenced by their levels of verbal behavior and to some extent by stimulus familiarity. The data are discussed in the context of alternative theoretical accounts of the processes believed to underlie equivalence.
\end{abstract}

There currently exists a well-established literature on demonstrations of derived equivalence relations in both adults and children (Carr, Wilkinson, Blackman, \& McIlvane, 2000; Devany, Hayes, \& Nelson, 1986). In his seminal work on equivalence class formation, Sidman (1971) attempted to establish equivalence relations between written words and pictures in an effort to train an adult with mental retardation to read. This was the first study to employ the matching-to-sample (MTS) methodology as a medium in which to generate derived equivalence performances (for an historical account of Sidman's research on equivalence, see his 1994 book). Although Sidman's early work involved a mentally retarded participant, the majority of equivalence studies since have concentrated primarily on demonstrations of equivalence in typically developing and verbally sophisticated adult participants. Indeed,

The authors thank two anonymous reviewers who provided kind and constructive commentaries on an earlier version of this article.

Correspondence regarding this article should be sent to Yvonne Barnes-Holmes, Department of Psychology, National University of Ireland Maynooth, Maynooth, Co. Kildare, Ireland. E-mail: Yvonne.barnes-holmes@nuim.ie 
only limited research has investigated the formation of equivalence classes in developmentally disabled populations (Carr et al., 2000; Devany et al., 1986; Eikeseth \& Smith, 1992).

One of the central issues that have been addressed in studies of equivalence with developmentally delayed populations is the role played by levels of verbal ability (Barnes, McCullagh, \& Keenan, 1990; Devany et al., 1986). In the study by Devany et al., the researchers compared the equivalence performances of normally developing preschoolers, developmentally delayed children with limited verbal abilities, and developmentally delayed children with little or no verbal abilities. The results of the study indicated that all of the verbally able children (both normally developing and developmentally delayed) readily demonstrated equivalence, compared with none of the children from the verbally unskilled group. The researchers argued that their findings lent support to the view that language abilities covary, at least to some extent, with the ability to demonstrate equivalence. Barnes et al. (1990) reported similar results with children with hearing impairments.

One of the most notable attempts to account for the relationship between verbal abilities and equivalence was proposed by Horne and Lowe in their naming theory (Horne \& Lowe, 1996). According to this account, equivalence relations are mediated through bidirectional stimulus relations that merge between speaker and listener repertoires. That is, individuals see the object to be named, name the object (speaker response), hear the name of the object (listening response), and then orient toward the object. In this naming cycle, the bidirectional relations between the object and the name are indirectly established. Hence, according to naming theory, the bidirectional relations that constitute equivalence are mediated through naming relations.

An implicit assumption of naming theory is that equivalence formation will, at least, be hampered in the absence of naming, and Dugdale and Lowe (1990) provided some support for this view when they reported that children's weak equivalence outcomes could be improved with explicit name training. These findings received further support when Eikeseth and Smith (1992) reported using a common naming strategy prior to, and during, conditional discrimination training to successfully establish two three-member equivalence classes in high-functioning children with autistic spectrum disorder (ASD). Furthermore, in a study with normally developing 6-year-olds, Pilgrim, Jackson, and Galizio (2000) reported that instructed naming (Experimenter provided a name for each stimulus) generated more rapid derived equivalence performances than self-naming (participants encouraged to generate individualized name for each stimulus). While these latter findings raise questions about the means by which explicit naming strategies facilitate equivalence, they nonetheless offer further support to the view that naming is important in this regard.

As one would expect, counterevidence for naming theory has also emerged. For example, Carr et al. (2000) reported research involving persons aged between 13 and 19 years old who lacked significant naming skills but had limited repertoires of manual signing. The findings indicated that in spite of their naming deficits, 4 of the 5 participants successfully demonstrated equivalence, and thus the data ran counter to one of naming theory's basic assumptions. On closer inspection, however, the findings appear more equivocal, because participants had extensive MTS and equivalence training, and their verbal deficits may not have been as great as the researchers believed. 
In a series of studies of the relationship between naming and derived symmetry performances, Barnes-Holmes, Barnes-Holmes, Roche, and Smeets (2001a, 2001b) systematically compared the effects of explicit name training and multiple-exemplar training on derived symmetry performances with normally developing 4- and 5-year-olds. The results of several studies indicated that explicit exemplar training of the target symmetry relations produced quicker derived performances than explicit name training in the context of novel stimulus sets. These researchers argued that their findings were more in keeping with the emphasis on multiple-exemplar training suggested by relational frame theory (RFT) than the emphasis on naming suggested by naming theory.

According to RFT, naming behavior makes available large numbers of stimuli and responses (i.e., heard and spoken words) by which numerous examples of bidirectional responding may be explicitly trained. Specifically, RFT suggests that explicitly reinforced object-name and name-object relations in particular (characteristic of early natural language interactions) provide a history of explicit symmetry training. In this way, naming provides one important way in which the generalized operant of derived symmetry may be established across exemplars (see Barnes, 1994, 1996; Barnes \& Holmes, 1991; Barnes \& Roche, 1996a, 1996b). According to this view, therefore, naming does not produce symmetry directly but instead provides the type of history and some of the contextual cues that control the relational operant of symmetry. The theory argues that equivalence relations are established in a similar manner, as well as that a history of naming may enhance symmetry or equivalence responding but is neither necessary nor sufficient for such responding to occur (Hayes, 1996).

According to RFT, an individual's level of verbal competence should correlate positively with the ability to derive arbitrary relations, such as equivalence, but this relationship may not necessarily be mediated by naming abilities (as would be predicted by naming theory). Specifically, naming theory predicts that naming should function as a powerful intervention for remediating deficits in derived relations such as equivalence. In contrast, RFT predicts that exemplar training, rather than naming, is the critical feature. Unfortunately, the existing behavioral literature contains relatively limited empirical analyses of the relationships among naming ability, verbal competence, and derived relational responding.

\section{The Current Research}

The empirical work reported here was an attempt to address relationships among naming, verbal competence, and equivalence by comparing the equivalence performances of children with autism categorized with different levels of verbal ability. It is important to emphasize at this point that the differential levels of verbal competence for the children was of greater significance, for current experimental purposes, than the fact that they had been diagnosed with ASD. However, because of the difficulty in determining the relationship between their language deficits and their other ASD features, we attempted to select a sample of children with a similar diagnosis and a broadly similar behavioral profile.

The research also had three secondary aims that were concerned with the putative roles of stimulus nameability, familiarity, and verbal 
antecedents on equivalence class formation. The concern with nameability derived directly from comparisons between predictions made by naming theory and RFT. Put simply, would unnamed stimuli come to participate in equivalence classes, and if so, would this participation occur with ease similar to that of nameable stimuli? A positive association between nameability and equivalence would offer some support to naming theory; a negative association would seem to favor RFT. However, such theoretical comparisons were not of primary concern; hence the issue was more about the nature of the relationship between naming and equivalence in the context of the current procedures.

The concern with familiarity emerged from findings reported by Holth and Arntzen (1998) in which equivalence performances with familiar stimuli were superior to those with unfamiliar stimuli. Furthermore, equivalencetraining programs frequently commence MTS training with familiar stimuli and systematically decrease familiarity as training progresses. Hence, in the current context it was possible that stimulus familiarity would exert an inadvertent influence over equivalence that could easily be attributed to naming.

Our concerns with the potential role of verbal antecedents resulted from pilot work in which strong equivalence and related performances of several children appeared to be dependent to some extent on the provision of specific antecedents. Furthermore, a strong feature of the CABAS (Comprehensive Application of Behavior Analysis to Schooling) training, to which all participants here were exposed as part of their daily education, involves the systematic use of antecedents. Thus we wanted to determine the extent to which the target equivalence performances here may have been dependent on the use of antecedents.

\section{Experiment 1}

\section{Methods}

\section{Participants}

Eight children (referred to as Participants 1-8) took part in Experiment 1. All had been independently diagnosed with ASD, and one or more of the following clinical tools had been used to formulate these diagnoses: the British Ability Scales, the Gilliam Autism Rating Scales, and the Vineland Adaptive Behavior Scales. All of the children attended a CABAS (Comprehensive Application of Behavior Analysis to Schooling) facility (see Greer, 2002) in either Cork or Dublin (Ireland). As part of the CABAS system, the children were classified in terms of their verbal capabilities (see Greer \& Ross, 2007 for a comprehensive review of the evolution of verbal capacity milestones and independence). The four broad areas of verbal capacity to which the participants here had been assigned were Listener/Prespeaker, Speaker/Prereader, Speaker/Reader, and Reader/Writer. The gender, age, and categorized level of verbal ability of each participant are presented in Table 1. Table 2 provides a description of the verbal capabilities of each of the relevant categories according to the CABAS system. All of the children had a history of MTS training through their education at CABAS. 
Table 1

Gender, Age, and Categorized Level of Verbal Ability of Each Participant in Experiment 1

\begin{tabular}{ccccc}
\hline Participant & Gender & Autism Spectrum & $\begin{array}{c}\text { Age } \\
\text { (years:months) }\end{array}$ & $\begin{array}{c}\text { Level of } \\
\text { Verbal Ability }\end{array}$ \\
\hline P1 & Male & Mild & $5: 5$ & Reader/Writer \\
P2 & Male & Mild & $6: 3$ & \\
\hline P3 & Male & Moderate & $5: 4$ & \\
P4 & Male & Moderate & $6: 7$ & Speaker/Reader \\
P5 & Female & Moderate & $5: 10$ & Speaker/ \\
P6 & Male & Moderate & $6: 4$ & Prereader \\
P7 & Female & Moderate & $5: 6$ & Listener/ \\
P8 & Male & Moderate & $6: 10$ & \\
\hline
\end{tabular}

Table 2

Summary of Levels of Verbal Capability for Participants according to Greer and Ross (2007)

Reader/Writer

Can use written text to provide themselves with useful, entertaining, and necessary responses to setting events and environmental contingencies

Can use verbal material without time constraints that govern and control listener-speaker exchanges

Individual may control environmental contingencies through mediation of the reader (either close by or in remote location)

Children at this level will have mastered the skills at the earlier levels of speaker and listener capabilities

Speaker/Reader

Conversational units

Can function as a listener to their own verbal behavior

Self-talk

Naming

Learning early reading skills (discrimination of text, text comprehension)

Speaker/Prereader Can use listener in their environment to mediate their needs

Sequelics

Can respond to intraverbals such as impure mands and tacts

Children can respond to questions in mand, tact, or intraverbal functions

As listeners they are reinforced by speaker responses

Engages in interlocking verbal operants

Listener/Prespeaker

Perform verbally governed behavior-basic directions

Comply with instructions and track tasks (first, then)

Generalized imitation

Attention to task or individual (speaker)

Visual tracking

Matching repertoire

Listener component of naming 


\section{Setting and Materials}

All aspects of Experiment 1 were conducted in a CABAS $^{\circledR}$ classroom during normal teaching hours (thus, five other students and five teachers were also present in the room during each session). However, neither the other students nor the teachers interacted with a participant during any part of an experimental session. Each participant sat at a desk with the Experimenter seated beside him or her. All of the experimental trials were presented in tabletop format. Eight sets of stimuli were employed in Experiment 1. Each stimulus set consisted of two classes, each with three members (i.e., six class members in each set). This yielded a total of 16 stimulus classes. For experimental purposes, each stimulus class member was referred to using an alphanumeric label, with related alphanumerics for the three stimuli in each class (e.g., A1, B1, C1; A2, B2, C2; and so on). Participants never saw these labels. The stimulus classes designated for use at each experimental stage are presented in Table 3.

Table 3

Stimulus Classes Designated for Each Stage in Experiment 1

\begin{tabular}{|c|c|c|c|}
\hline \multicolumn{4}{|c|}{$\begin{array}{l}\text { Stage } 1 \\
\text { Nameable and familiar stimuli with antecedent }\end{array}$} \\
\hline \multicolumn{2}{|c|}{ Set 1} & \multicolumn{2}{|c|}{ Set 2} \\
\hline $\mathrm{A} 1$ & A2 & A3 & A4 \\
\hline B1 & B2 & B3 & B4 \\
\hline $\mathrm{Cl}$ & $\mathrm{C} 2$ & C3 & $\mathrm{C} 4$ \\
\hline \multicolumn{4}{|c|}{$\begin{array}{l}\text { Stage } 2 \\
\text { Unnameable and familiar stimuli with antecedent }\end{array}$} \\
\hline \multicolumn{2}{|c|}{ Set 3} & \multicolumn{2}{|c|}{ Set 4} \\
\hline A5 & A6 & A7 & A8 \\
\hline B5 & B6 & B7 & B8 \\
\hline $\mathrm{C} 5$ & C6 & $\mathrm{C} 7$ & $\mathrm{C} 8$ \\
\hline \multicolumn{4}{|c|}{$\begin{array}{l}\text { Stage } 3 \\
\text { Unnameable and unfamiliar stimuli with antecedent }\end{array}$} \\
\hline \multicolumn{2}{|c|}{$\begin{array}{l}\text { Set } 5 \\
\end{array}$} & \multicolumn{2}{|c|}{ Set 6} \\
\hline A9 & $\mathrm{A} 10$ & A11 & $\mathrm{A} 12$ \\
\hline B9 & B10 & B11 & B12 \\
\hline $\mathrm{C} 9$ & $\mathrm{C} 10$ & $\mathrm{C} 11$ & $\mathrm{C} 12$ \\
\hline \multicolumn{4}{|c|}{$\begin{array}{l}\text { Stage } 4 \\
\text { Unnameable and unfamiliar stimuli with no antecedent }\end{array}$} \\
\hline \multicolumn{2}{|c|}{ Set 7} & \multicolumn{2}{|c|}{ Set 8} \\
\hline $\mathrm{A} 13$ & $\mathrm{~A} 14$ & $\mathrm{~A} 15$ & A16 \\
\hline B13 & B14 & B15 & B16 \\
\hline $\mathrm{C} 13$ & $\mathrm{C} 14$ & $\mathrm{C} 15$ & $\mathrm{C} 16$ \\
\hline
\end{tabular}

To manipulate stimulus nameability and familiarity, Sets 1-4 were composed of written words, actual objects, and pictures, whereas Sets 5-8 contained abstract printed symbols and written nonsense syllables. 
Each three-member class consisted of a written word (always referred to using the alphanumeric $A$ ); an object (always referred to using the alphanumeric $B$ ); and a picture (always referred to using the alphanumeric $C)$, and thus comprised word-object-picture relations. The A stimuli were either actual words (Sets 1-4) or nonsense syllables (Sets 5-8) and were always printed in black Arial 100 on a white card (approx. size, 36 in.2). The B stimuli were always real objects (Sets 1-4 included a banana and a stapler) or abstract objects (Sets 5-8 included a shape made up from toilet tissue rolls glued together). The $C$ stimuli were always photographs (Sets 1-4) or printed sketches (Sets 5-8). The nameability or familiarity of the stimuli was manipulated systematically across the four stages of the experimental sequence.

\section{Experimental Sequence}

The study consisted of four experimental stages conducted as Stages $1,2,3$, and 4, respectively. Each of the four stages was almost identical in format and contained the same four experimental phases (Phases 1, 2, 3, and 4 conducted in that order). Phase 1 involved name training and testing to determine whether the participants could name the objects employed as $\mathrm{B}$ stimuli. Phase 2 always involved explicit A-B training, and Phase 3 involved explicit B-C training. Phase 4 consisted of an equivalence test of the $\mathrm{A}-\mathrm{C}$ and $\mathrm{C}-\mathrm{A}$ relations to be derived from the conditional discrimination training in Phases 2 and 3. In Stage 1 all trials in all phases involved the Experimenter providing a vocal antecedent. Each of the four experimental phases involved training and testing with two stimulus sets (see Table 3), and participants were presented with both sets before they proceeded to the next stage. Participants could proceed from one stage to another only once they had successfully passed the equivalence test for both stimulus sets in the previous stage.

The stimuli employed in Stage 2 differed from those used in Stage 1 in that they were familiar but unnamed. Stage 3 was identical to Stage 2, except that the stimuli employed were unfamiliar and unnamed. Stage 4 was identical to Stage 3, except that no vocal antecedent was provided for any of the training or test trials.

\section{General Procedure}

The amount of training and testing to which each child was exposed depended on his or her performance during each experimental phase and stage. Each child was exposed to one experimental session 3 to 4 days per week (availability permitting). On average, each child underwent approximately eight experimental sessions. Each session lasted approximately $20 \mathrm{~min}$, with a break of several minutes at an appropriate point approximately halfway through the session. If at any point during training or testing, a child asked or indicated the desire to stop, the experimental trials were terminated for that session, and the same trials were repeated in the following session.

Corrective feedback. Corrective feedback was provided after all training trials but after none of the test trials. Corrective feedback for correct responses consisted of verbal praise on a reinforcement schedule of fixed ratio 1 plus the presentation of edibles on a variable ratio 3 schedule. During the presentation of verbal praise for a correct response, the Experimenter said "Good girl/boy, well done" (or words to that effect) and the next trial 
was presented immediately. Edibles were presented at the same time as the verbal praise so that the two events occurred simultaneously after the child's response had been emitted. If a child emitted an incorrect response, the Experimenter repeated the antecedent (if appropriate) and modeled the correct response (e.g., by touching the correct comparison stimulus). No verbal feedback consequated an incorrect response. The next trial was then presented immediately. Edibles were not provided for incorrect responding. This pattern of trial repetition and correction was in keeping with the CABAS instructional programs with which the children were familiar.

Specific contingencies were in place for various forms of on-task behavior throughout the study. That is, participants received verbal praise in the form of "nice sitting" or "nice being quiet" for the maintenance of on-task behaviors during the sessions. All feedback or reinforcement for on-task behavior was presented only after the stimuli had been removed from the table, and at least $30 \mathrm{~s}$ had elapsed since the end of the last test trial.

Phase 1: Name-training/testing. Phase 1 normally consisted of four nametesting trials for each of the two B stimuli from the two stimulus sets currently in use during any one stage (e.g., B1, B2, B3, and B4 were tested during Stage 1; see Table 3). As an aside, in the current research we tested and trained the names only of the B stimuli, rather than of all three sets of $\mathrm{A}, \mathrm{B}$, and $\mathrm{C}$ stimuli. We did so to avoid the provision of a common name to A, B, and C, which might make all three stimuli functionally equivalent irrespective of the conditional discrimination training, and to avoid giving all three a separate name and thus potentially interfering with the derivation of equivalence among the target stimuli. Explicit naming of the middle stimulus (B in this case) therefore seemed to be the simplest and safest of the three stimuli with which naming could be manipulated.

The name-testing trials were conducted in pairs, with two trials for each set. At the beginning of each trial, the two objects from each set (e.g., B1 and B2 from Set 1) were placed on the experimental table in front of the child. The Experimenter then selected one of the objects and simply held it up in front of the child for naming (the children's established histories in CABAS precluded the need to provide an instruction for naming). A naming response was recorded as correct if the child correctly named the object within $10 \mathrm{~s}$ of its selection. Failure to emit any response was recorded as incorrect. After the trial the Experimenter replaced the object on the table. The next naming test trial was presented immediately and involved selecting the other object on the table. The two remaining test trials involved the B stimuli from the second set and were conducted in the same way. Participants who responded correctly to all four naming test trials in Phase 1 were deemed to have passed the naming test, and they then proceeded immediately to Phase 2 .

The name testing described above was conducted during all four experimental stages. However, because familiar and nameable stimuli were necessary for Stage 1, it was important to ensure that all participants passed the naming test at this stage. If a participant failed to respond correctly to any of the naming test trials in Phase 1 of Stage 1 , he or she was immediately exposed to explicit name training to ensure that all of the objects to be employed as B stimuli during that stage could be named correctly. The explicit name training to which participants were exposed was based on specific name-training procedures employed as part of the 
CABAS instructional program. This form of training consisted of two parts: echoic training and independent name training. During Part 1, an echoic response was established in the presence of the object. During these trials, the Experimenter held up the target object and presented the name (e.g., the Experimenter held up the banana and said "banana"), to which the child was required to emit the correct echoic response ("banana"). If a child failed to emit any response within $10 \mathrm{~s}$ of the presentation of the item, this was recorded as incorrect. Verbal praise was provided after each correct echoic response, whereas the name was simply repeated by the Experimenter on those trials in which a correct response was not emitted. During echoic name training, each child was required to produce five consecutively correct echoic responses for each object before proceeding to independent name training with that object. Immediately after the echoic name training, the child was provided with trials involving independent name training in which the Experimenter no longer provided the name, and the child was required to name the object independently upon its selection. The same corrective feedback was provided during these trials, and participants were required to produce 20 consecutively correct independent tacts before proceeding to Phase 2 . This echoic and independent name-training sequence was conducted for each of the four objects in Sets 1 and 2 from Stage 1 that the participants had failed to name independently on the initial naming test. This explicit name training was conducted only in Stage 1.

Phase 2: A-B training. An MTS many-to-one procedure was used to train all of the conditional discriminations. Participants were first exposed to explicit training of the A-B relations (e.g., A1-B1 and A2-B2). Prior to each trial, the two comparison stimuli (e.g., B1: a banana, and B2: a stapler) were placed on the table to the left and right below the sample (e.g., A1-the printed word BANANA). At the beginning of each trial, the Experimenter touched the sample stimulus and immediately presented the antecedent "Goes With." The Experimenter then remained looking at the sample for $10 \mathrm{~s}$ (but the B stimuli remained in the Experimenter's visual field) during which the child was required to make a response. During this time, the Experimenter did not look at the child or otherwise interact with the child in any way. A correct response involved the child indicating the correct B stimulus, either by pointing to it or picking it up. Corrective feedback and edibles were provided as described above. Both A-B trial types were identical, and each was presented randomly. Across each of two exposures to each trial type, the locations of the comparison stimuli were counterbalanced. Participants were required to reach a mastery criterion of eight consecutively correct A-B responses before proceeding to Phase 3 . Hence the amount of A-B training to which each participant was exposed depended entirely on the number of trials required by each child to reach the mastery criterion in this phase.

Phase 3: B-C training. Explicit training of the B-C relations (e.g., B1-C1 and B2-C2) was identical to the A-B training, and participants once again were required to reach a mastery criterion of eight consecutively correct responses on the B-C relations before proceeding with the experiment.

Phase 4: Equivalence testing. After reaching the mastery criterion on the $\mathrm{BC}$ relations, participants were immediately exposed to a test for combined symmetry and transitivity involving the $\mathrm{A}-\mathrm{C}$ and $\mathrm{C}-\mathrm{A}$ relations. This testing comprised four basic trial types (e.g., A1-C1, A2-C2, C1-A1, and C2-A2). Each participant underwent five exposures to each of the four trial types 
randomly presented across a block of 20 test trials. Once again, the locations of the comparisons were counterbalanced across trials. Breaks between experimental sessions never occurred between the training and testing phases with a particular stimulus set. The equivalence testing in Phase 4 was identical to the conditional discrimination training in Phases 2 and 3, except that no corrective feedback was presented after any test trials. If a participant emitted an incorrect response to a test trial, the trial was terminated by removal of the stimuli and the next test trial was presented immediately. All feedback or reinforcement for on-task behavior was presented only after the stimuli had been removed from the table, and at least $30 \mathrm{~s}$ had elapsed since the end of the last test trial.

To pass the equivalence test, participants were required to produce 16 of 20 correct responses, with no more than one error occurring on each of the four trial types. If a participant failed to pass the equivalence test at this point, he or she was reexposed to the conditional discrimination training in Phase 2, involving the same stimulus set. The participant was then reexposed to the equivalence test. This cycle of training and testing continued until all participants had passed the equivalence test for each stimulus set. Once the equivalence test for the first stimulus set in any one stage had been passed, participants repeated the entire process with the second set. It was only after both sets had been completed that a participant could proceed to the next experimental stage. The completion of the equivalence test with the second stimulus set in Stage 4 marked the end of the experiment for all of the children.

Interobserver agreement. Twenty-five percent of all test trials and 30\% of all training trials were recorded by an independent observer who was at the opposite end of the experimental table from the participant and Experimenter. There was 100\% agreement on all trials observed by both the Experimenter and the independent observer.

\section{Results}

The dependent variables on which the children were assessed were levels of accuracy during the equivalence tests (see Table 4) and the number of training trials required to meet criterion during the conditional discrimination training phases (see Table 5). The data for each of these measures are presented separately.

\section{Equivalence Accuracy Data}

The data suggest that the number of reexposures to equivalence was greater for the more difficult stimulus sets. Specifically, only 1 participant (P8) required multiple exposures to Stage 1; 3 required reexposure to Stage 2 (P8, P7, and P6); 4 required reexposure to Stage 3 (P8, P6, P5, and P3); and 3 had repeated exposures in Stage 4 (P6, P4, and P3). Furthermore, the need for repeated test exposures across subsequent stages appeared to be influenced by levels of verbal behavior. Specifically, the majority of repeated test exposures, particularly on the later stimulus sets, were conducted with the children with the lowest levels of verbal behavior. For example, P8 (Listener/ Prespeaker) required several exposures to Stages 1, 2, and 3; P6 (Speaker/ Prereader) required multiple exposures in Stages 2, 3 and 4; and yet P1 (Reader/Writer) required no reexposure to any set. 
Table 4

Number of Correct Responses and Exposures on Equivalence Tests Across the Four Experimental Stages for Participants Categorized at Each of the Four Levels of Verbal Behavior

\begin{tabular}{|c|c|c|c|c|c|c|c|c|c|}
\hline \multirow{3}{*}{$\begin{array}{l}\text { Levels of } \\
\text { Verbal } \\
\text { Behavior }\end{array}$} & \multirow[b]{3}{*}{ Participant } & \multicolumn{8}{|c|}{ Experimental Stage and Stimulus Set } \\
\hline & & \multicolumn{2}{|c|}{ Stage 1} & \multicolumn{2}{|c|}{ Stage 2} & \multicolumn{2}{|c|}{ Stage 3} & \multicolumn{2}{|c|}{ Stage 4} \\
\hline & & Set 1 & Set 2 & Set 3 & Set 4 & Set 5 & Set 6 & Set 7 & Set 8 \\
\hline \multirow{2}{*}{$\begin{array}{l}\text { Reader/ } \\
\text { Writer }\end{array}$} & $\mathrm{P} 1$ & 20 & 19 & 20 & 20 & 19 & 16 & 19 & 19 \\
\hline & $\mathrm{P} 2$ & 20 & 19 & 20 & 20 & 20 & 20 & 19 & 19 \\
\hline \multirow{8}{*}{$\begin{array}{l}\text { Speaker/ } \\
\text { Reader }\end{array}$} & \multirow{4}{*}{ P3 } & 19 & 19 & 20 & 18 & 15 & 18 & 17 & 20 \\
\hline & & - & - & - & - & 18 & - & 6 & 19 \\
\hline & & - & - & - & - & - & - & 8 & - \\
\hline & & - & - & - & - & - & - & 20 & - \\
\hline & \multirow{2}{*}{ P4 } & 20 & 19 & 19 & 17 & 17 & 18 & 15 & 20 \\
\hline & & - & - & - & - & - & - & 19 & - \\
\hline & \multirow{2}{*}{ P5 } & 19 & 18 & 19 & 17 & 6 & 18 & 17 & 19 \\
\hline & & - & - & - & - & 17 & - & - & - \\
\hline \multirow{5}{*}{$\begin{array}{l}\text { Speaker/ } \\
\text { Prereader }\end{array}$} & \multirow{3}{*}{ P6 } & 16 & 18 & 17 & 17 & 19 & 16 & 14 & 16 \\
\hline & & - & - & - & 18 & - & 17 & 18 & 20 \\
\hline & & - & - & - & - & - & 19 & - & - \\
\hline & \multirow{2}{*}{ P7 } & 18 & 18 & 18 & 19 & 16 & 19 & 18 & 19 \\
\hline & & - & - & 19 & - & - & - & - & - \\
\hline \multirow{3}{*}{$\begin{array}{l}\text { Listener/ } \\
\text { Prespeaker }\end{array}$} & \multirow{3}{*}{ P8 } & 16 & 16 & 15 & 19 & 18 & 18 & 13 & 15 \\
\hline & & 11 & 19 & 18 & - & - & - & 13 & 18 \\
\hline & & 17 & - & - & - & - & - & 17 & - \\
\hline
\end{tabular}

Note. Dashed lines (-) indicate that reexposure to a particular stimulus set was not necessary (i.e., participant had passed the test). Multiple cells presented for each set indicate that multiple exposures to that set were necessary to pass the test.

\section{Conditional Discrimination Training Data}

The total numbers of training trials required by each participant at each stage, as well as the overall mean number of training trials required by the children in the four levels of verbal behavior are presented in Table 5. The table indicates that the children from the higher levels of verbal ability appeared to require less training to reach criterion on the conditional discriminations than children from the lower ability groups. Specifically, the mean number of trials for the Reader/Writers across all four stages was 37 (minimum was 32), compared with a mean of 140 for the Listener/Prespeakers. Somewhere between these two were the midrange children, who required 77 (Speaker/ Readers) and 82 (Speaker/Prereaders) trials to reach criterion. These data suggest that the children's levels of verbal behavior influenced the ease with which they proceeded through the conditional discrimination training.

Table 5 also indicates the mean number of trials required by the children overall to complete training across all four stages. Specifically, a mean total of 108 training trials were required for the children to complete Stage 4, 
relative to only 69 for Stage 1 . However, only minor overall differences in means were recorded among Stages 1, 2, and 3. Stage 4 required more training to demonstrate positive equivalence outcomes, probably because of the removal of the vocal antecedent. In the CABAS system, children are exposed to extensive instructional histories in which vocal antecedents (often identical to that employed here) set the occasion for responding and thus their absence renders the children more uncertain about what will follow.

Table 5

Number of Training Trials Required Across the Four Experimental Stages for Each Participant from the Four Levels of Verbal Behavior

\begin{tabular}{|c|c|c|c|c|c|c|c|c|c|c|}
\hline \multirow{3}{*}{$\begin{array}{l}\text { Levels of } \\
\text { Verbal } \\
\text { Behavior }\end{array}$} & \multirow[b]{3}{*}{ Participant } & \multicolumn{8}{|c|}{ Experimental Stage and Stimulus Sets } & \multirow{3}{*}{$\begin{array}{l}\text { Mear } \\
\text { Trials }\end{array}$} \\
\hline & & \multicolumn{2}{|c|}{ Stage 1} & \multicolumn{2}{|c|}{ Stage 2} & \multicolumn{2}{|c|}{ Stage 3} & \multicolumn{2}{|c|}{ Stage 4} & \\
\hline & & Set 1 & Set 2 & Set 3 & Set 4 & Set 5 & Set 6 & Set 7 & Set 8 & \\
\hline \multirow{2}{*}{$\begin{array}{l}\text { Reader/ } \\
\text { Writer }\end{array}$} & $\mathrm{P} 1$ & 32 & 34 & 32 & 32 & 71 & 38 & 36 & 39 & \multirow{2}{*}{37} \\
\hline & P2 & 32 & 33 & 32 & 32 & 35 & 38 & 33 & 48 & \\
\hline \multirow{3}{*}{$\begin{array}{c}\text { Speaker/ } \\
\text { Reader }\end{array}$} & P3 & 47 & 33 & 33 & 56 & 169 & 54 & 303 & 90 & \multirow{3}{*}{77} \\
\hline & P4 & 94 & 55 & 47 & 34 & 51 & 63 & 114 & 59 & \\
\hline & P5 & 32 & 79 & 50 & 37 & 145 & 49 & 79 & 65 & \\
\hline \multirow{2}{*}{$\begin{array}{l}\text { Speaker/ } \\
\text { Prereader }\end{array}$} & P6 & 101 & 62 & 51 & 103 & 50 & 191 & 180 & 102 & \multirow{2}{*}{82} \\
\hline & P7 & 41 & 65 & 105 & 48 & 43 & 43 & 49 & 83 & \\
\hline $\begin{array}{l}\text { Listener/ } \\
\text { Prespeaker }\end{array}$ & P8 & 250 & 111 & 130 & 50 & 76 & 65 & 254 & 187 & 140 \\
\hline $\begin{array}{l}\text { Mean } \\
\text { Trials }\end{array}$ & & \multicolumn{2}{|c|}{69} & \multicolumn{2}{|c|}{55} & \multicolumn{2}{|c|}{74} & \multicolumn{2}{|c|}{108} & \\
\hline
\end{tabular}

In summary, the equivalence test performances appeared to be influenced by both the children's levels of verbal behavior and the degree of familiarity and nameability of the stimuli. Specifically, children from the higher levels of verbal ability required fewer exposures to pass the equivalence test than those from the lower levels of ability. Furthermore, the amount of reexposure to the tests appears to have increased when the stimuli presented were less familiar and less namable. Similar patterns of responding were observed in the context of the conditional discrimination training. In general, the children from the higher verbal levels required less training than those in the lower levels, and to some extent more training was necessary when the stimuli were less familiar and less nameable.

\section{Discussion}

One important issue raised by the findings from Experiment 1 is the possibility that the results obtained arose, at least in part, by the sequence of training and testing to which the participants were exposed. In others words, if the experimental stages were reversed, would similar patterns of responding be observed? This issue was addressed in Experiment 2. 


\section{Experiment 2}

Experiment 2 was identical in format to Experiment 1, except that the sequence of stages was reversed. Specifically, the children were first exposed to Stage 4, then Stage 3, then Stage 2, and finally Stage 1. As a result, the children were first exposed to the abstract and unnameable stimuli without an antecedent (Stage 4), and only at the end were they exposed to the familiar and nameable stimuli with an antecedent.

\section{Methods}

\section{Participants}

Seven experimentally naïve children participated in Experiment 2. All had independent diagnoses of ASD and were matched by level of verbal behavior with the participants from Experiment 1, with the exception that Experiment 2 did not contain a child categorized as a Listener/Prespeaker. The gender, age, and categorized level of verbal ability of each participant are presented in Table 6. Once again, all children had a history of MTS training through their education at CABAS.

Table 6

Gender, Age, and Categorized Level of Verbal Ability of Each Participant in Experiment 2

\begin{tabular}{ccccc}
\hline Participant & Gender & Autism Spectrum & $\begin{array}{c}\text { Age } \\
\text { (years:months) }\end{array}$ & $\begin{array}{c}\text { Level of } \\
\text { Verbal Ability }\end{array}$ \\
\hline P9 & Male & Mild & $6: 1$ & Reader/Writer \\
P10 & Male & Moderate & $5: 6$ & \\
\hline P11 & Male & Mild-Moderate & $5: 10$ & \\
P12 & Female & Moderate & $6: 3$ & Speaker/Reader \\
P13 & Female & Mild & $7: 5$ & \\
\hline P14 & Male & Moderate & $5: 11$ & Speaker/Prereader \\
P15 & Male & Moderate & $8: 6$ & \\
\hline
\end{tabular}

\section{Setting and Materials}

All aspects of the setting were identical to Experiment 1 . All of the stimuli from the previous study were employed again in Experiment 2, with the addition of a novel Set 9 for P15. This new stimulus set also comprised two three-member classes (i.e., A17-B17-C17 and A18-B18-C18). All six stimuli in Set 9 were abstract, unfamiliar, and unnameable. The two A stimuli (A17 and A18) were printed nonsense words (i.e., WEP and JOM); the B stimuli (B17 and B18) were black painted abstract objects constructed by the Experimenter from waste materials; and the C stimuli (C17 and C18) were abstract symbols printed on white cards. Once again, the $\mathrm{C}$ stimuli bore no obvious physical similarity to either the A or B stimulus, thus rendering the two classes arbitrary. A range of small edible items, including small parts of sweets and potato chips, were also employed as reinforcers for correct responding. 


\section{Procedure}

All procedural aspects of Experiment 2 were identical to Experiment 1, except for the reversal of the four stages. That is, participants were exposed to the following sequence: Stage 4 followed by Stage 3 followed by Stage 2, and finally Stage 1.

\section{Corrective Feedback}

All aspects of corrective feedback were identical to Experiment 1, and the same reinforcement schedules for correct responding during training (but not testing) were employed with Ps 9-15.

\section{Interobserver Agreement}

Twenty-five percent of all test trials and 30\% of all training trials were recorded by an independent observer who was at the opposite end of the experimental table from the participant and Experimenter. There was $100 \%$ agreement on all trials observed by both the Experimenter and the independent observer.

\section{Results}

\section{Equivalence Accuracy Data}

The children's levels of accuracy and number of exposures on the equivalence tests in Experiment 2 are presented in Table 7. No child required multiple exposures to the equivalence tests during Stage 1, 1 child during Stage 2, 6 during Stage 3, and 5 during Stage 4 (see Table 7). These numbers are larger than those recorded in the previous experiment and indicate that even children with high levels of verbal ability required multiple exposures to pass the equivalence tests in this context. Of course, the current sequence undoubtedly rendered Experiment 2 simply more difficult than Experiment 1, but that in itself suggests that the familiarity and nameability of the stimuli influenced the equivalence performances. There was also some evidence again that the need for repeated test exposures was influenced by the children's levels of verbal behavior. Specifically, P15 (Speaker/Prereader) required no less than 27 extra exposures to the equivalence tests, compared with P9 (Reader/ Writer), who required none. The numbers of repeated exposures necessary for the children in the intervening verbal ability groups were mixed.

\section{Conditional Discrimination Training Data}

The total numbers of training trials required by each participant at each stage, as well as the overall mean number of training trials required by each of the four verbal ability groups, are presented in Table 8 . Once again, the children from the higher levels of verbal ability appeared to require less training to reach criterion on the conditional discriminations than children from the lower ability groups. Specifically, the mean number of trials for the Reader/Writers across all four stages was 57, compared with a mean of 104 for the Speaker/Prereaders. The mean performances of the Speaker/Readers were identical to those of the Reader/Writers. These data suggest that once 
again the children's levels of verbal behavior influenced the ease with which they proceeded through the conditional discrimination training, especially where the levels of verbal behavior were lowest.

\section{Table 7}

Number of Correct Responses and Exposures on Equivalence Tests Across the Four Experimental Stages for Participants Categorized at Each of the Four Levels of Verbal Behavior

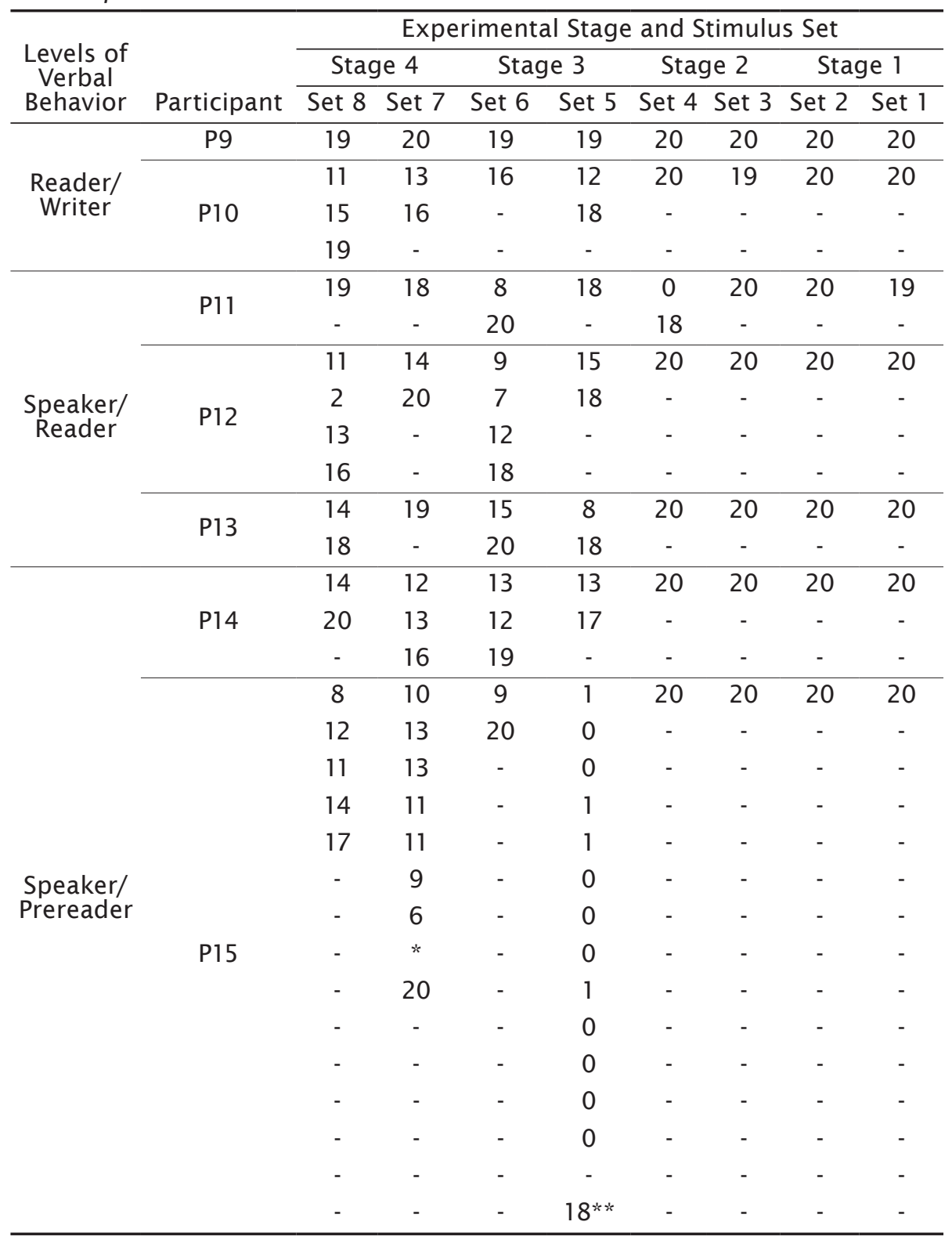

* Testing on this set of stimuli was terminated.

** Testing with stimulus Set 5 was terminated, and training and testing with Set 9 were introduced. 
The table also indicates the mean number of trials required by the children overall to complete training across all four stages. Specifically, a mean total of 110 training trials were required for the children to complete Stage 4, relative to only 32 for Stage 1. Unlike Experiment 1, the mean numbers for Stages 1 and 2 were almost identical but very much smaller than the mean numbers for Stages 3 and 4 (the latter were in fact identical to one another). The sizeable shift in training needs between Stages 3 and 2 suggests that familiarity in particular may have been critical to the ease of learning the conditional discriminations in Experiment 2.

\section{Table 8}

Number of Training Trials Required Across the Four Experimental Stages for Each Participant from the Four Levels of Verbal Behavior

\begin{tabular}{|c|c|c|c|c|c|c|c|c|c|c|}
\hline \multirow{3}{*}{$\begin{array}{l}\text { Levels of } \\
\text { Verbal } \\
\text { Behavior }\end{array}$} & \multirow[b]{3}{*}{ Participant } & \multicolumn{8}{|c|}{ Experimental Stage and Stimulus Sets } & \multirow{3}{*}{$\begin{array}{l}\text { Mean } \\
\text { Trials }\end{array}$} \\
\hline & & \multicolumn{2}{|c|}{ Stage 4} & \multicolumn{2}{|c|}{ Stage 3} & \multicolumn{2}{|c|}{ Stage 2} & \multicolumn{2}{|c|}{ Stage 1} & \\
\hline & & Set 8 & Set 7 & Set 6 & Set 5 & Set 4 & Set 3 & Set 2 & Set 1 & \\
\hline \multirow{2}{*}{$\begin{array}{l}\text { Reader/ } \\
\text { Writer }\end{array}$} & P9 & 60 & 39 & 38 & 35 & 32 & 32 & 32 & 32 & \multirow{2}{*}{57} \\
\hline & P10 & 200 & 146 & 80 & 39 & 47 & 36 & 32 & 32 & \\
\hline \multirow{3}{*}{$\begin{array}{l}\text { Speaker/ } \\
\text { Reader }\end{array}$} & P11 & 98 & 59 & 65 & 38 & 79 & 33 & 32 & 32 & \multirow{3}{*}{57} \\
\hline & P12 & 134 & 68 & 144 & 72 & 34 & 32 & 32 & 32 & \\
\hline & P13 & 73 & 48 & 64 & 64 & 32 & 32 & 32 & 32 & \\
\hline \multirow{2}{*}{$\begin{array}{l}\text { Speaker/ } \\
\text { Prereader }\end{array}$} & P14 & 114 & 73 & 81 & 92 & 32 & 32 & 32 & 32 & \multirow{2}{*}{104} \\
\hline & P15 & 183 & 248 & 84 & $\begin{array}{c}(462) \\
56^{*}\end{array}$ & 37 & 36 & 32 & 32 & \\
\hline $\begin{array}{l}\text { Mean } \\
\text { Trials }\end{array}$ & & \multicolumn{2}{|c|}{110} & \multicolumn{2}{|c|}{101} & \multicolumn{2}{|c|}{38} & \multicolumn{2}{|c|}{32} & \\
\hline
\end{tabular}

* The number in parentheses represents the total number of training trials within the first set of stimuli; the number outside parentheses is the total number of training trials when the stimuli were changed.

In summary, therefore, the findings from Experiment 2 were generally similar to those from Experiment 1, in terms of the impact of level of verbal behavior and stimulus difficulty on equivalence. However, the considerable problems that surrounded the children's exposure to the abstract stimuli first in the absence of an antecedent make it difficult to clearly determine the influence of the other factors.

\section{Discussion}

One issue raised by the findings from both studies, however, concerned the extent to which the data were specific to the children's diagnoses of developmental delay. Perhaps the patterns recorded thus far are not indicative of the influences of verbal ability and stimulus familiarity or 
nameability on the equivalence performances of normally developing children but are specific to children from the designated population? One might ask, therefore, whether a similar pattern of responding would be recorded as part of a more natural developmental sequence. To address this issue, a sample of typically developing children was exposed to the same procedures in Experiment 3.

\section{Experiment 3}

\section{Method}

\section{Participants}

Three typically developing children (P16, P17, and P18) participated in Experiment 3. Their gender and ages are presented in Table 9. The children were recruited on the basis that they had not been identified by parents or teachers as presenting any learning difficulties. All 3 children attended the same national school in the Dublin area.

Table 9

Gender and Age of Each Participant in Experiment 3

\begin{tabular}{ccc}
\hline Participant & Gender & $\begin{array}{c}\text { Age } \\
\text { (years:months) }\end{array}$ \\
\hline P16 & Female & $7: 3$ \\
P17 & Male & $9: 2$ \\
P17 & Male & $10: 4$ \\
\hline
\end{tabular}

\section{Materials and Setting}

The participants completed the study individually in a quiet room in their school with only the Experimenter present. The same stimulus sets from Experiments 1 and 2 were employed in Experiment 3, with the exception of Sets 3 and 4 previously used in Stage 2. Two different familiar and unnameable stimulus sets replaced Sets 3 and 4 during Stage 2, because the verbally sophisticated participants could readily name the stimulus sets that had been used previously with the children with ASD.

\section{Procedure}

All procedural aspects of Experiment 3 were identical to those in Experiment 2.

\section{Interobserver Agreement}

Twenty-five percent of all test trials and 30\% of all training trials were recorded by an independent observer who was at the opposite end of the experimental table from the participant and Experimenter. There was $100 \%$ agreement on all trials observed by both the Experimenter and the independent observer. 


\section{Results}

\section{Equivalence Accuracy Data}

The children's levels of accuracy and number of exposures on the equivalence tests in Experiment 3 are presented in Table 10. All 3 children proceeded relatively quickly through the equivalence tests. None required multiple exposures to the tests during Stage 1 or 2. During Stage 3, 1 child (P16) required two exposures, and during Stage 4, 1 child (P18) required three exposures. These data indicate that the normally developing children produced strong equivalence performances overall, with weaknesses recorded only during those stages in which abstract stimuli were employed.

Table 10

Number of Correct Responses and Exposures on Equivalence Tests Across the Four Experimental Stages for Participants in Experiment 3

\begin{tabular}{|c|c|c|c|c|c|c|c|c|}
\hline \multirow[b]{3}{*}{ Participant } & \multicolumn{8}{|c|}{ Experimental Stage and Stimulus Set } \\
\hline & \multicolumn{2}{|c|}{ Stage 4} & \multicolumn{2}{|c|}{ Stage 3} & \multicolumn{2}{|c|}{ Stage 2} & \multicolumn{2}{|c|}{ Stage 1} \\
\hline & Set 8 & Set 7 & Set 6 & Set 5 & Set 10 & Set 11 & Set 2 & Set 1 \\
\hline & 18 & 15 & 19 & 20 & 20 & 20 & 20 & 20 \\
\hline \multirow[t]{2}{*}{ P16 } & - & 0 & - & - & - & - & - & - \\
\hline & - & 19 & - & - & - & - & - & - \\
\hline \multirow{2}{*}{$\mathrm{P} 17$} & 20 & 18 & 2 & 20 & 20 & 20 & 20 & 20 \\
\hline & - & - & 20 & - & - & - & - & - \\
\hline P18 & 20 & 20 & 20 & 20 & 20 & 20 & 20 & 20 \\
\hline
\end{tabular}

\section{Conditional Discrimination Training Data}

The total numbers of training trials required by each participant at each stage, as well as the overall mean number of training trials required, are presented in Table 11. The training data recorded with the normally developing children demonstrated relatively rapid progression through training (i.e., the mean number of trials across all four stages was 38), with the largest numbers required during the abstract stages (e.g., P16 required 108 trials with Set 7 of Stage 4).

In summary, therefore, the findings from Experiment 3 were generally similar to those from Experiments 1 and 2. The data show that the verbally sophisticated children proceeded quickly through all four stages in terms of both conditional discrimination training and equivalence, with elevated numbers of training or test trials primarily required when abstract stimuli were employed. 
Table 11

Number of Training Trials Required Across the Four Experimental Stages for Each Participant in Experiment 3

\begin{tabular}{|c|c|c|c|c|c|c|c|c|c|}
\hline \multirow[b]{3}{*}{ Participant } & \multicolumn{8}{|c|}{ Experimental Stage and Stimulus Sets } & \multirow{3}{*}{$\begin{array}{l}\text { Mean } \\
\text { Trials }\end{array}$} \\
\hline & \multicolumn{2}{|c|}{ Stage 4} & \multicolumn{2}{|c|}{ Stage 3} & \multicolumn{2}{|c|}{ Stage 2} & \multicolumn{2}{|c|}{ Stage 1} & \\
\hline & Set 8 & Set 7 & Set 6 & Set 5 & Set 4 & Set 3 & Set 2 & Set 1 & \\
\hline P16 & 32 & 108 & 36 & 33 & 32 & 32 & 32 & 32 & \\
\hline $\mathrm{P} 17$ & 63 & 33 & 65 & 32 & 32 & 32 & 32 & 32 & 38 \\
\hline P18 & 32 & 32 & 32 & 32 & 32 & 32 & 32 & 32 & \\
\hline Mean Trials & \multicolumn{2}{|c|}{50} & \multicolumn{2}{|c|}{38} & \multicolumn{2}{|c|}{32} & \multicolumn{2}{|c|}{32} & \\
\hline
\end{tabular}

\section{General Discussion}

The current research comprised three experiments across which normally developing children and children with autism were provided with conditional discrimination training and equivalence tests involving stimuli with varying degrees of nameability and familiarity. The children were distinguished primarily in terms of their levels of verbal ability, with the prediction that children with the most competent verbal skills would produce more rapid equivalence outcomes than those children with less developed verbal repertoires. The findings from Experiments 1 and 2 demonstrated that the children with autism with different levels of verbal competence produced differential equivalence outcomes in terms of test accuracy and the numbers of test exposures necessary. Specifically, those children with higher levels of verbal ability (e.g., Reader/Writers) produced more rapid equivalence performances than those with lower verbal skills (e.g., Listener/Prespeakers). Furthermore, the equivalence outcomes recorded with the latter children were necessarily preceded by greater numbers of training trials to reach criterion on the conditional discrimination training. The data from Experiment 3 supported the former findings when the typically developing children (with presumably high levels of verbal competence) proceeded rapidly through the training and testing. Indeed, relatively little difference could be identified between these children and those who had been diagnosed as autistic but who displayed high levels of verbal ability. This concordance between the two groups suggests that the training and test performances targeted here were at least in part mediated by the children's verbal abilities.

The current study attempted to systematically identify the role of stimulus nameability across Stages 1 and 2 to determine the impact of naming as a specific feature of verbal competence on equivalence outcomes. The findings recorded across the three studies indicated minor superiority in performance between Stage 1, involving nameable stimuli, and the latter stages, in which the stimuli did not have clearly identifiable names. Although this outcome might suggest that stimulus nameability enhanced the training and testing performances, it is difficult to rule out the possible influence of stimulus familiarity or the 
presence of an antecedent, both of which were systematically manipulated in the latter stages. Of critical importance, therefore, are differences between Stage 1 and Stage 2, where nameability was the only distinction. However, the data recorded here show strong performances overall for both stages and only very minor differences between them, thus suggesting a limited impact for stimulus naming.

Indeed, central to the naming issue are the positive equivalence outcomes of P8 in Experiment 1, whose verbal capabilities were categorized only as Listener/Prespeaker. These findings support those of Carr et al. (2000), who also showed that participants with minimal verbal ability were capable of demonstrating equivalence, albeit with much more training. Taken together, this concordance of evidence indicates that individuals at this level of verbal ability can demonstrate equivalence, even with unnameable stimuli, thus providing further support for RFT's predictions regarding the utility of multiple-exemplar training.

However, the data reported here may be interpreted equally in favor of one theory over the other. For example, superiority of performance in Stage 1 relative to the latter stages may be deemed consistent with Naming Theory, and it remains possible that the children were generating their own names for the stimuli presented in the latter stages. In contrast, it may also be argued that the data are consistent with RFT, because of the theory's emphasis on exemplar training. The current studies systematically employed exemplar training using multiple stimulus sets to build up competence during the training and testing performances, and the findings generally show that positive outcomes emerged. Although one might conclude that these outcomes were a direct result of the exemplar training and testing, the studies did not incorporate, for example, a multiple baseline design that would permit closer examination of the relationship between the exemplar training and the equivalence outcomes. Nonetheless, the findings are consistent with existing empirical evidence that demonstrates the utility of exemplar training in the facilitation or establishment of derived relations such as equivalence (Barnes-Holmes et al., 2001a, 2001b). The question, however, regarding the superiority of one account over the other cannot be fully addressed here.

The current research also attempted to examine the impact of stimulus familiarity on the training and testing of equivalence. Although it is difficult to separate the issue of familiarity from the primary aims of the study (such as level of verbal ability and nameability), the data show notable differences in training and test performances between Stages 2 and 3, where stimulus familiarity was the primary distinction. Indeed, the use of familiar stimuli appeared to correlate with more rapid training and test performances with many of the children, autistic and typically developing. Furthermore, stimulus familiarity was seen to specifically enhance the performances of P15 in Experiment 2 when this child repeatedly failed to pass the equivalence test with unfamiliar stimuli. Overall, the data suggest that the use of familiar stimuli facilitated the training of conditional discriminations and the production of equivalence. Nonetheless, it is difficult to determine the precise ways in which the positive impact of familiarity may have occurred. For example, one might argue that the use of familiar stimuli allowed participants to more readily generate names for the stimuli, and thus familiarity enhanced performance through a form of covert naming. Such a hypothesis cannot be ruled out here, although there was no evidence at the time that such naming behavior was present. It may 
also be the case that familiarity enhanced performance, because these stimuli participated in existing stimulus classes such as "stapler" participating in an equivalence class with "dad's work." In this latter case, the participation of the stimuli in existing classes may have facilitated their participation in new classes. The current study, however, was not designed to determine the utility of either hypothesis, and future research would be necessary to address this issue directly.

The current study was partially concerned with the effect of a vocal antecedent on the emergence of equivalence performances. The results indicated that participants demonstrated some difficulties when the vocal antecedent ("goes with") was initially removed. Although the removal of the antecedent in this case was coupled with other variables such as stimulus familiarity, it is reasonable to assume that the absence of the antecedent impacted negatively on the target performance. However, it is possible that the specific content of the antecedent (i.e., the actual words "goes with") had less control over the behavior than the presence of an antecedent per se. In other words, any phrase may have functioned as an antecedent and the words "goes with" were not specifically necessary. There is some empirical evidence in support of this perspective from the view that MTS itself provides a context for matching.

The autistic children in the current study were selected from special educational facilities, all of which relied heavily on MTS training procedures. As a result, it is difficult to determine here whether the specific content of the current antecedent or the presence of any verbal antecedent was critical to the behavior. However, the impact of the antecedent was greater when the sequence of training the stages was reversed, and thus when the stimuli were initially unfamiliar and unnameable. In this case, the absence of stimulus familiarity and nameability perhaps rendered the child more dependent on the antecedent than when these variables were present. Thus one might conclude that the importance of the antecedent and its control over behavior is sensitive to the broader context.

Theresults of the current study would appear to have considerable educational significance for children with autism. There were specific and systematic variations in the training and testing conducted during the experiments that provide some insight into the variables that impacted on the children's conditional discrimination training and equivalence outcomes. Although it is impossible to generalize across children from any given population, these variables may provide a useful focus for relevant educational or remediation programs. Furthermore, in the context of equivalence and its importance in a great many learning programs, the identification of critical variables that facilitate the learning path and which might facilitate the broader operant of derivation per se would be significant. The impact of such variables on the design and implementation of a myriad of teaching and remediation programs could impact greatly on future development of educational programs and assessment.

\section{References}

BARNES, D. (1994). Stimulus equivalence and relational frame theory. The Psychological Record, 44, 91-124.

BARNES, D. (1996). Naming as a technical term: Sacrificing behavior analysis at the altar of popularity. Journal of the Experimental Analysis of Behavior, 65, 264-267. 
BARNES, D., \& HOLMES, Y. (1991). Radical behaviorism, stimulus equivalence, and human cognition. The Psychological Record, 41, 19-31.

BARNES, D., MCCULLAGH, P. D., \& KEENAN, M. (1990). Equivalence class formation in non-hearing impaired children and hearing impaired children. Analysis of Verbal Behavior, 8, 1-11.

BARNES, D., \& ROCHE, B. (1996). Relational frame theory and stimulus equivalence are fundamentally different: A reply to Saunders. The Psychological Record, 46, 236-256.

BARNES, D., \& ROCHE, B. (1996). Stimulus equivalence and relational frame theory are fundamentally different: A reply to Saunders' commentary. The Psychological Record, 46, 489-507.

BARNES-HOLMES, Y., BARNES-HOLMES, D., ROCHE, B., \& SMEETS, P. M. (2001a). Exemplar training and a derived transformation of function in accordance with symmetry. The Psychological Record, 51, 287-308.

BARNES-HOLMES, Y., BARNES-HOLMES, D., ROCHE, B., \& SMEETS, P. M. (2001b). Exemplar training and a derived transformation of function in accordance with symmetry: II. The Psychological Record, 51, 589-604.

CARR, D., WILKINSON, K. M., BLACKMAN, D., \& MCILVANE, W. J. (2000). Equivalence classes in individuals with minimal verbal repertoires. Journal of the Experimental Analysis of Behavior, 74, 101-114.

DEVANY, J. M., HAYES, S. C., \& NELSON, R. O. (1986). Equivalence class formation in language-able and language-disabled children. Journal of the Experimental Analysis of Behavior, 46, 243-257.

DUGDALE, N., \& LOWE, C. F. (1990). Naming and stimulus equivalence. In D. E. BLACKMAN \& H. LEJEUNE (Eds.), Behavior analysis in theory and practice: Contributions and controversies (pp. 115-138). London: Erlbaum.

EIKESETH, S., \& SMITH, T. (1992). The development of functional and equivalence classes in high-functioning autistic children: the role of naming. Journal of the Experimental Analysis of Behavior, 58, 123-133.

GREER, R. D. (2002). Designing teaching strategies: An applied behavior analysis systems approach. San Diego, CA: Academic Press.

GREER, R. D., \& ROSS, D. E. (2007). Verbal behavior analysis: Inducing and expanding new verbal capabilities in children with language delays. Boston: Pearson Press.

HAYES, S. C. (1996). Developing a theory of derived stimulus relations. Journal of the Experimental Analysis of Behavior, 65, 309-311.

HOLTH, P., \& ARNTZEN, E. (1998). Stimulus familiarity and the delayed emergence of stimulus equivalence or consistent non-equivalence. The Psychological Record, 48, 81-110.

HORNE, P. J. \& LOWE, C. F. (1996). On the origins of naming and other symbolic behavior. Journal of the Experimental Analysis of Behavior, 65, $185-241$.

PILGRAM, C., JACKSON, J., \& GALIZIO, M. (2000). Acquisition of arbitrary conditional discriminations by young normally developing children. Journal of the Experimental Analysis of Behavior, 73, 177-193.

SIDMAN, M. (1971). Reading and auditory-visual equivalences. Journal of Speech and Hearing Research, 14, 5-13.

SIDMAN, M. (1994). Equivalence relations and behavior: A research story. Boston: Authors Cooperative. 